\title{
Expression of tumour necrosis factor receptors by bronchoalveolar cells in hypersensitivity pneumonitis
}

\author{
B. Chen*, Z. Tong*, Q. Ye*, S. Nakamura*, U. Costabel* and J. Guzman",
}

ABSTRACT: Tumour necrosis factor receptors (TNFR) and the Fas receptor (FasR) have been implicated in the pathogenesis of interstitial lung diseases. The current authors examined the expression of TNFR-1, TNFR-2 and FasR by bronchoalveolar cells in hypersensitivity pneumonitis (HP).

Cell surface receptor expression on bronchoalveolar lavage cells was analysed by immunocytochemistry in $11 \mathrm{HP}$ patients, 11 idiopathic pulmonary fibrosis (IPF) patients and 10 controls.

TNFR-1, TNFR-2 and FasR were expressed on a higher percentage of alveolar macrophages (AM) in HP compared with controls and IPF patients. TNFR-2 and FasR expression on lymphocytes was also higher in HP than in controls and in IPF. TNFR-1, TNFR-2 and FasR expression correlated positively with the percentage of lymphocytes, and negatively with the percentage of AM in HP. Expression of TNFR-1 on AM and TNFR-2 on lymphocytes correlated with the percentage of neutrophils in HP.

In conclusion, this study shows evidence of altered expression of tumour necrosis factor superfamily receptors in hypersensitivity pneumonitis.

KEYWORDS: Alveolar lymphocytes, alveolar macrophages, Fas receptors, hypersensitivity pneumonitis, tumour necrosis factor receptors 1 and 2

$\mathbf{T}$ umour necrosis factor (TNF)- $\alpha$ is a proinflammatory cytokine, produced mainly by activated monocytes/macrophages, and exerts its function by binding to its cognate tumour necrosis factor receptor (TNFR)-1 and TNFR-2 on the cell surface. This has been implicated in the pathogenesis of a number of inflammatory lung diseases [1, 2]. The biological effects elicited by TNF- $\alpha$ include cytotoxicity, a role in endotoxic shock, and inflammatory, immunoregulatory, proliferative and antiviral responses [3].

The TNFR superfamily is a group of cell-surface receptors crucially involved in the maintenance of homeostasis of the immune system. These receptors can lead to either apoptosis or survival of immune cells when ligated by their corresponding ligands [4]. Some studies have shown the involvement of apoptotic receptors in interstitial lung diseases $[5,6]$.

The TNF receptors, TNFR-1 (p55/60, CD120a) and TNFR-2 (p75/80, CD120b), can exist as membrane-associated or soluble proteins. The soluble receptors possess a TNF-neutralising ability [1]. TNFR-1 shares homology with Fas and contains a death domain in the cytoplasmic tail capable of transducing a death signal. TNFR2 does not contain a death domain in its cytoplasmic tail; its role during TNF-mediated signal transduction is considered to be indirect in that it appears to aid in the recruitment of TNF to the cell membrane and passes the signal to TNFR-1 [7], or regulates the amount of TNF that is accessible to TNFR-1 [8]. The TNF receptors can lead to either apoptosis or survival. TNF and Fas-mediated apoptosis is induced via the activation of caspases. TNF-mediated anti-apoptosis involves TNFR-associated factor-2, which indirectly binds to TNFR-1 through the TNF receptor-associated death domain and the receptor-interacting protein or directly binds to TNFR-2. This activates the transcription nuclear factor- $\kappa \mathrm{B}$, which triggers inflammation and also plays an important role in regulating apoptosis $[9,10]$.

The Fas receptor (FasR; Apo1/CD95) may mediate apoptosis in susceptible target cells when bound to the Fas ligand (FasL) or to agonistic anti-Fas antibodies [11]. Soluble isoforms of the Fas molecule have been identified, which can block apoptosis induced by FasL in vitro [12].

\section{AFFILIATIONS}

*Dept of Pneumology and Allergy, Ruhrlandklinik, Medical Faculty, University of Essen, Essen, and "General and Experimental Pathology, Ruhr University, Bochum, Germany.

\#Dept of General Medicine and Clinical Investigation, Nara Medical University, Nara, Japan.

\section{CORRESPONDENCE}

U. Costabel

Ruhrlandklinik

Tüschener Weg 40

45239 Essen

Germany

Fax: 492014334029

E-mail: eri.costabel@t-online.de

Received:

July 162004

Accepted after revision:

February 042005

\section{SUPPORT STATEMENT}

This work was supported by the Ichihiro Kanehara Foundation, Chihiro and Kiyoko Yokochi Fund (Japan), AFPR (Arbeitsgemeinschaft zur Förderung der Pneumologie an der Ruhrlandklinik; Germany), and FORUM (Ruhr University, Bochum, Germany). 
Dysregulation of the Fas system, leading to accelerated Fasmediated death, has been implicated in diseases. In contrast, deficient Fas-mediated apoptosis, e.g. by genetic alteration of the receptor or the ligand, has been shown to lead to a syndrome of lymphoproliferation and auto-immunity in mouse strains and in patients $[13,14]$.

The expression of TNFR-2 and FasR was found to be increased on bronchoalveolar lavage (BAL) lymphocytes in sarcoidosis and hypersensitivity pneumonitis (HP), and also on circulating T-cells from the peripheral blood in both diseases [5]. In contrast, expression of TNFR-1 was not observed on BAL lymphocytes in both diseases, and only minor expression was reported on blood lymphocytes in HP [5]. The expression of TNFR-1 and FasR on BAL macrophages was noted to be increased in sarcoidosis [6]. These two receptors were found to be upregulated on synovial tissue cells in rheumatoid arthritis compared with peripheral blood mononuclear cells (PBMC) [15]. Upregulated expression of FasR was described on peripheral T-cells in patients with systemic lupus erythematosus (SLE) [16]. Thus, it has been suggested that the expression of these three receptors is involved in the inflammatory immune response.

In the pathogenesis of $\mathrm{HP}$, alveolitis has been linked to numerous endogenous mediators, especially TNF- $\alpha$. To test whether increased expression of TNFR-1, TNFR-2 and FasR is present in the inflammatory response of $\mathrm{HP}$, the current authors evaluated the expression of these three receptors by alveolar cells in patients with HP, in comparison with patients with idiopathic pulmonary fibrosis (IPF) and control subjects.

\section{MATERIALS AND METHODS \\ Study population}

In total, 11 consecutive patients with HP (mean \pm SEM age $51.4 \pm 3.4$ yrs) were investigated (table 1 ). All were untreated at the time of BAL. They were lavaged 0-4 days after the last antigen exposure. Seven were budgerigar fanciers, two bird fanciers and two had humidifier's lung. Of these, three

\begin{tabular}{|c|c|c|c|}
\hline & HP & IPF & Controls \\
\hline Female/male & $4 / 7$ & $2 / 9$ & $4 / 6$ \\
\hline Age yrs & $51.4 \pm 3.4^{\#}$ & $70.7 \pm 1.5^{\circ}$ & $51.3 \pm 5.4$ \\
\hline Nonsmokers & 11 & 3 & 4 \\
\hline Current smokers & 0 & 0 & 2 \\
\hline Ex-smokers & 0 & 8 & 4 \\
\hline VC \% pred & $68.3 \pm 5.1$ & $73.2 \pm 5.4$ & $86.2 \pm 3.9$ \\
\hline TLC \% pred & $75.8 \pm 6.0$ & $66.7 \pm 2.5^{\circ}$ & $91.8 \pm 3.5$ \\
\hline FEV $_{1} /$ VC \% & $80.1 \pm 3.2$ & $80.9 \pm 3.0$ & $73.7 \pm 3.2$ \\
\hline $\mathrm{Pa}, \mathrm{O}_{2} \mathrm{mmHg}$ & $78.0 \pm 3.8$ & $69.3 \pm 1.5$ & $77.4 \pm 3.8$ \\
\hline $\mathrm{PA}_{\mathrm{A}-\mathrm{a}, \mathrm{O}_{2}} \mathrm{mmHg}$ & $22.0 \pm 3.0$ & $31.3 \pm 2.0$ & $27.1 \pm 4.5$ \\
\hline
\end{tabular}

Data are presented as $n$ and mean \pm SEM. HP: hypersensitivity pneumonitis; IPF: idiopathic pulmonary fibrosis; VC: vital capacity; TLC: total lung capacity; FEV1: forced expiratory volume in one second; $\mathrm{Pa}, \mathrm{O}_{2}$ : arterial oxygen tension; $\mathrm{PA}-\mathrm{a}, \mathrm{O}_{2}$ : alveolar-arterial oxygen tension difference. $\#: p<0.01$ (HP versus IPF); $\mathrm{p}<0.01$ (HP or IPF versus controls). $\mathrm{kPa}=\mathrm{mmHg} \times 0.133$. presented with the acute disease, and eight with the chronic form of insidious outset. All patients fulfilled the following diagnostic criteria: 1) a history of exposure to organic antigens; 2) clinical signs and symptoms consistent with HP; 3) radiological features and/or functional abnormalities characteristic of interstitial lung disease; 4) evidence of serum precipitins against one or more organic antigens; and 5) BAL fluid with increased lymphocytes. The manifestations on high resolution computed tomography (HRCT) of the eight patients with chronic form showed widespread, dominant groundglass densities, with only minor reticular shadowing and no honeycombing. Late-stage cases with extensive fibrosis were not investigated in this study.

In total, 11 consecutive patients with IPF (aged $70.7 \pm 1.5 \mathrm{yrs}$ ) were included. They all fulfilled the recently published American Thoracic Society/European Respiratory Society criteria, including the HRCT characteristics for the diagnosis of IPF [17]. None had left ventricular cardiac failure or a history of chronic pulmonary infections.

The control subjects consisted of 10 patients (aged $51.3 \pm 5.4 \mathrm{yrs}$ ). They had no evidence of interstitial lung disease and no history of exposure to antigens known to cause HP. They underwent diagnostic bronchoscopy for various reasons (mediastinal abnormalities, pleural disease, suspicion of recurrent tuberculosis that was not confirmed, exclusion of sarcoidosis or other interstitial lung disease) and had a normal BAL cytology.

Written, informed consent was obtained from the patients according to institutional guidelines.

\section{Bronchoalveolar lavage}

BAL was performed by instilling a total volume of $200 \mathrm{~mL}$ of sterile isotonic saline in $10 \times 20-\mathrm{mL}$ aliquots into the right middle or left lingular lobe via a fibreoptic bronchoscope with immediate aspiration by gentle suction after each aliquot. A volume of $>50 \%$ was retrieved. The recovered BAL fluid was filtered through two layers of sterile gauze and subsequently centrifuged at $500 \times g$ for $10 \mathrm{~min}$ at $4^{\circ} \mathrm{C}$. The cells were counted in a haemocytometer. Cell viability was assessed by Trypan blue dye exclusion and the cell number was counted by a standard haemocytometer. Cell differentials were made on smears stained with May-Grünwald-Giemsa by counting 600 cells.

\section{Immunocytochemical analysis}

The monoclonal antibodies (MAb) used in this analysis included CD3, CD4 and CD8 (Dako, Copenhagen, Denmark), FasR (Upstate Biotechnology Incorporated, Lake Placid, NY, USA), TNFR-1 (Bender MedSystems, Vienna, Austria), and TNFR-2 (Serotec Ltd, Oxford, UK). The peroxidase-antiperoxidase (PAP) method was applied to identify membrane antigens on the freshly recovered BAL cells, as previously described [18]. Briefly, $10 \mathrm{~mL}$ cell suspension $\left(5 \times 10^{6}\right.$ cell $\left.\cdot \mathrm{mL}^{-1}\right)$ was added to the reaction areas of adhesion slides (Bio-Rad, Munich, Germany) for $10 \mathrm{~min}$. After the cells had settled on the glass surface, they were incubated with MAb for $15 \mathrm{~min}$ and fixed with $0.05 \%$ glutaraldehyde for $5 \mathrm{~min}$, then washed three times with HEPES-buffered washing solution containing $\mathrm{NaCl}$ and $\mathrm{KCl}$. Cells were then incubated with a 
gelatine-containing medium supplemented with $10 \%$ swine serum and $0.2 \%$ bovine serum albumin for $15 \mathrm{~min}$ to prevent nonspecific binding of immunoglobulin (Ig) to glass and cells. Subsequently, the cells were incubated with rabbit anti-mouse IgG, then with swine anti-rabbit IgG and, finally, with the rabbit PAP immunocomplex, each incubation lasting for $5 \mathrm{~min}$ (all reagents from Dako). Diaminobenzidine was used as substrate to visualise the reaction, and $\mathrm{OsO}_{4}$ for post-fixation. Specificity of the immunostaining was determined by omitting the primary $\mathrm{MAb}$ on a reaction area and using mouse IgG instead. No cross-reaction was observed. To evaluate the percentage of positive cells, $\geqslant 200$ macrophages or lymphocytes were counted under a light microscope.

\section{Statistical analysis}

All data were expressed as mean \pm SEM. The differences between HP and controls or IPF were compared using a Mann-Whitney Rank Sum test (for nonparametric data) or an unpaired t-test (for parametric data). The correlation of different parameters was analysed by Spearman rank order correlation coefficients. A level of $\mathrm{p}<0.05$ was accepted as statistically significant.

\section{RESULTS}

\section{Bronchoalveolar lavage fluid cell findings}

As shown in table 2, patients with HP had significant increases in the total cell number, the percentage of lymphocytes and mast cells, a significant decrease in the percentage of macrophages compared with controls and IPF, and significant increases in neutrophils and eosinophils only in comparison with controls. There were significant differences in the proportions of macrophages, eosinophils and neutrophils between IPF and controls.

\section{Expression of TNFR-1, TNFR-2 and FasR on BAL cells}

The percentages of TNFR-1+ alveolar macrophage (AM), TNFR-2+ AM and FasR+ AM were much higher in HP than in controls and IPF patients (all $\mathrm{p}<0.01$; fig 1a). The percentage of TNFR-2+ AM was lower in IPF than in controls $(p<0.05)$. TNFR-1 was not expressed on BAL lymphocytes. The percentages of TNFR-2+ and FasR+ BAL lymphocytes were markedly elevated in HP in comparison with controls and IPF


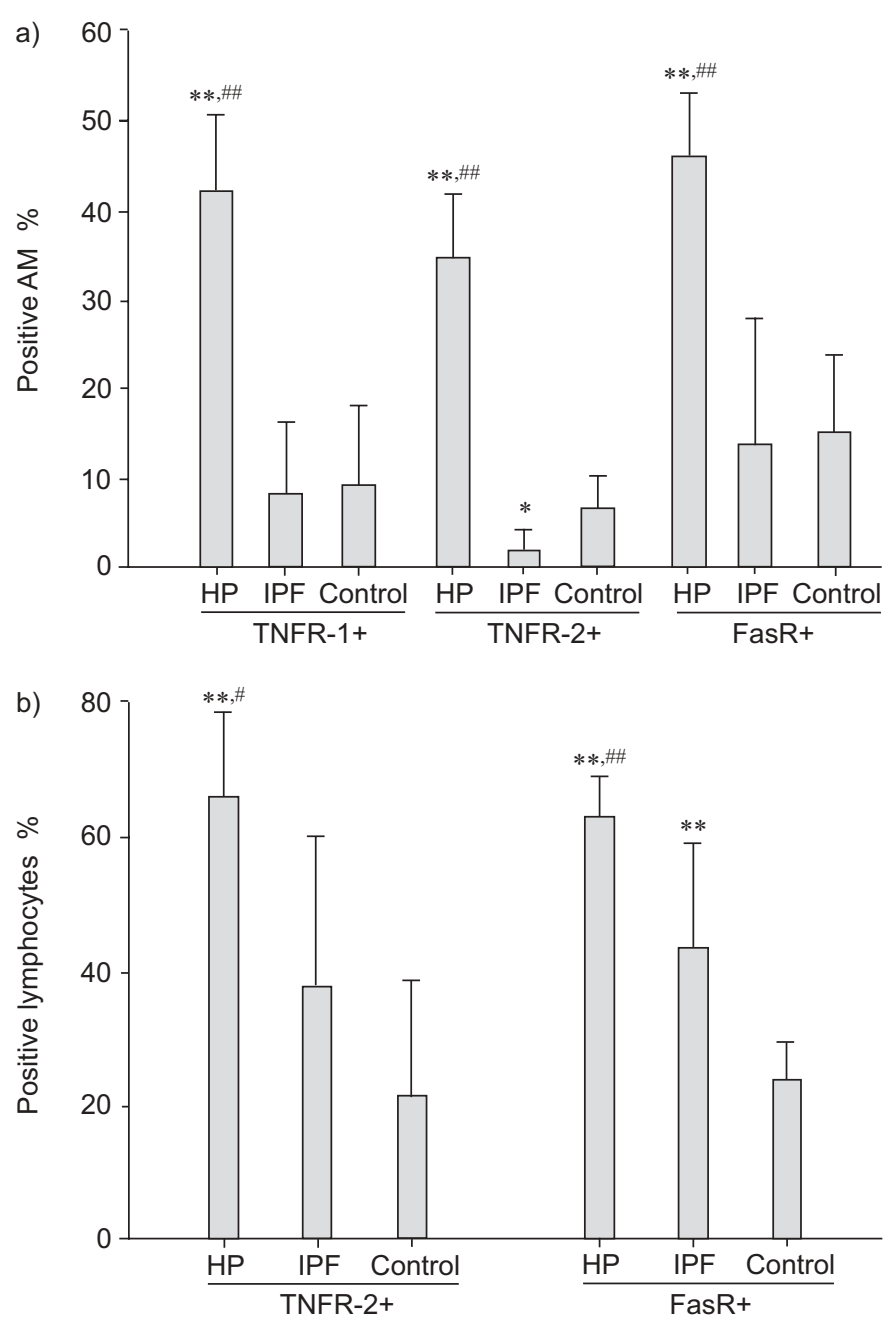

FIGURE 1. a) Expression of tumour necrosis factor receptor (TNFR)-1, TNFR-2 and Fas receptor (FasR) on alveolar macrophages (AM) in hypersensitivity pneumonitis (HP), idiopathic pulmonary fibrosis (IPF) and controls. b) Expression of TNFR-2 and FasR on bronchoalveolar lavage lymphocytes in HP, IPF and controls. Data are expressed as mean \pm SEM. ${ }^{*}: p<0.05$; ${ }^{* *}: p<0.01$ (HP or IPF versus control); $\#$ : $p<0.05$; \#\#: $p<0.01$ (HP versus IPF).

(fig 1b). In IPF, the FasR was expressed by a significantly higher percentage of BAL lymphocytes than in controls.

\section{Correlation between TNFR-1+, TNFR-2+ and FasR + BAL cells and other BAL parameters}

As shown in table 3, close correlations were found between the proportion of FasR+ AM and the proportion of TNFR-1+ AM and TNFR-2+ AM, as well as between TNFR-1+ AM and TNFR-2+ $\mathrm{AM} \quad(\mathrm{r}=0.84, \mathrm{p}<0.001 ; \mathrm{r}=0.7, \mathrm{p}=0.015 ; \mathrm{r}=0.6$, $\mathrm{p}=0.047$, respectively). The expression of TNFR-1, TNFR-2 and FasR correlated positively with the percentage of lymphocytes $(\mathrm{r}=0.72, \mathrm{p}=0.01 ; \mathrm{r}=0.8, \mathrm{p}=0.001 ; \mathrm{r}=0.87$, $\mathrm{p}<0.001$, respectively), and negatively with the percentage of macrophages in BAL of HP patients $(r=-0.83, \mathrm{p}<0.001 ; \mathrm{r}=$ $-0.76, p=0.006 ; r=-0.83, p<0.001$, respectively). The proportion of neutrophils in BAL of HP correlated with the expression of TNFR-1 on AM and TNFR-2 on BAL lymphocytes $(r=0.61$, $\mathrm{p}=0.04 ; \mathrm{r}=0.68, \mathrm{p}=0.02$, respectively). 


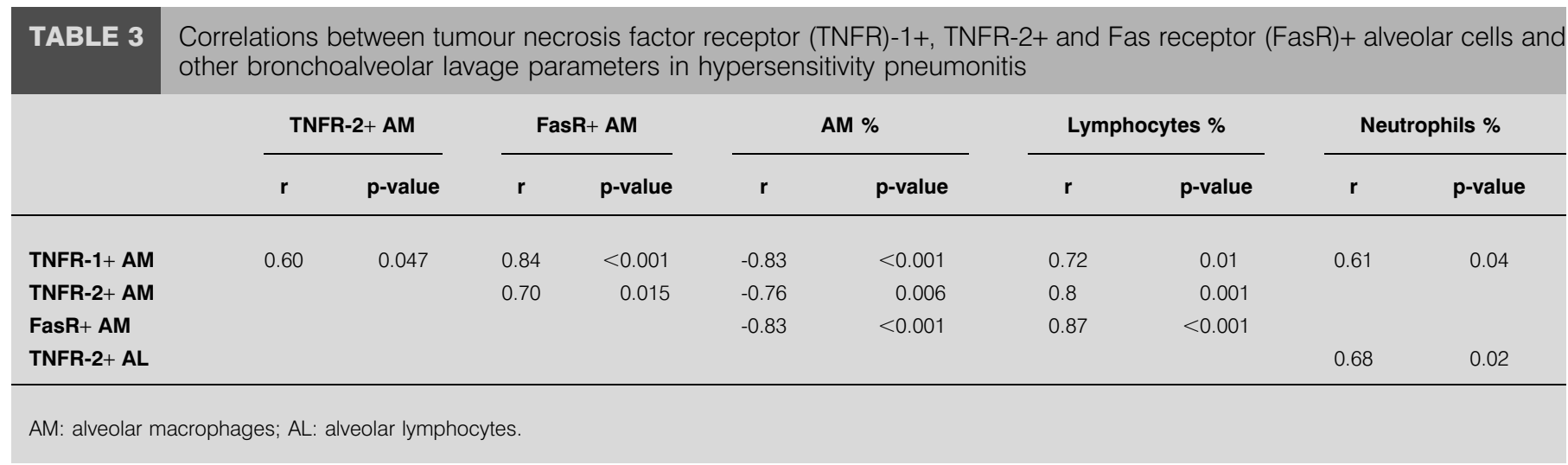

\section{DISCUSSION}

In this study, the current authors showed that the percentages of TNFR-1+ AM, TNFR-2+ AM and FasR+ AM, and TNFR-2+ and FasR+ BAL lymphocytes were markedly higher in HP patients in comparison with controls and IPF patients. There were positive correlations between the expression of TNFR-1 and TNFR-2 on AM in HP.

Recently, it has been shown that HP is characterised by a Thelper (Th)-1 type cytokine response, including increased release of interleukin (IL)-12 and IL-18 by AM [19, 20]. Numerous other proinflammatory cytokines, in particular TNF- $\alpha$ produced by macrophages, play a role in the inflammatory response of HP [2]. The role of TNF receptors in the pathogenesis of this disease is still poorly defined.

A previous study from the current authors revealed an increased expression of TNFR-1 and FasR on BAL macrophages of patients with sarcoidosis, which is another granulomatous lung disease [6]. The expression of TNFR-2 and FasR has been reported to be increased on BAL lymphocytes in sarcoidosis and HP [5]. In agreement with these data, GAEDE et al. [21] showed in a group of patients with various pulmonary disorders that TNFR-1 is predominantly expressed on AM and PBMC, and TNFR-2 was mainly found on AM, PBMC and BAL lymphocytes.

In a mouse model, TNFR-2 has been shown to trigger apoptosis of activated T-cells, and more than two-thirds of the apoptotic cells were found to be CD8+ T-cells [22]. TNFR-2deficient CD8+ T-cells are resistant to Fas/FasL-induced cell death [23]. TNF mediates the death of most CD8+ Tcells, whereas FasL mediates the death of most CD4+ T-cells [24, 25]. TNFR-2 can also signal lymphocyte activation, as shown for human thymocytes and peripheral T-cells stimulated by concavalin A [3]. In the current study, an increased expression of TNFR-2 on BAL lymphocytes in HP was found, which is characterised by intra-alveolar accumulation of CD8+ T-cells. TNFR-2 expression on such CD8+ T-cells in HP may trigger these cells to undergo apoptosis. Previously, it was shown that soluble TNFR-2 levels are increased in the BAL of HP [26], and it was suggested that this might serve as a negative feedback mechanism for the elimination and downregulation of activated T-cells within the alveolar space in HP in order to suppress the intense pulmonary inflammation.
Increased FasR expression on peripheral T-cells and PBMC has been found in sarcoidosis and HP, and on peripheral T-cells for SLE. This was discussed as a possible mechanism for the lymphopenia observed in SLE [5, 16]. FasR can also be expressed on AM in sarcoidosis [6] and on human blood eosinophils [11]. The present study revealed that the FasR expression on AM and BAL lymphocytes is enhanced in HP. The FasR expression on AM correlated positively with the expression of TNFR-1, TNFR-2 and the percentage of lymphocytes, and negatively with the percentage of AM in HP. A recent study demonstrated that circulating monocytes and tissue macrophages release TNF- $\alpha$ and IL- 8 following Fas ligation [27]. This indicates that Fas ligation can promote proinflammatory activity. It is conceivable that the increased expression of FasR plus TNFR-1 and TNFR-2 on AM may be indicators of the activation of AM and their proinflammatory activity, while the increased expression of FasR and TNFR-2 on BAL lymphocytes may be relevant to the occurrence of apoptosis of activated lymphocytes in the pulmonary microenvironment of HP.

In the present study, it was demonstrated that the expression of TNFR-1 closely correlated with the expression of TNFR-2 on $\mathrm{AM}$, and that both receptors correlated with the percentage of lymphocytes, and inversely with the percentage of macrophages in the BAL of HP patients. The increased expression of TNFR-1 and TNFR-2 on AM may reflect the local inflammatory activity of the lung. The current authors further found that the expression of TNFR-2 on BAL lymphocytes and of TNFR-1 on AM correlated with the percentage of neutrophils in the BAL of HP patients. Along these lines, a recent study showed that TNFR-1 facilitates neutrophil recruitment in BAL fluid after inhalation of lipopolysaccharide in mice [28]. Thus, TNFR-1 and TNFR-2 may have a function to recruit neutrophils to the local inflammatory site of the lung.

IPF is a chronic fibroproliferative disease, and suggested to be a Th2-mediated response. One study proposed that apoptosis of epithelial cells by Fas-FasL cross-linking is causally related to fibrosis [29]. The percentage of BAL lymphocytes expressing Fas were found to be higher in IPF than in controls, but lower than in HP. There were no differences in the expression of the other receptors on alveolar cells between IPF and controls, except for a lower proportion of TNFR-2+ AM in IPF than in controls. 
Taken together, this study demonstrates evidence for an increased expression of tumour necrosis factor receptor-1, tumour necrosis factor receptor-2 and Fas receptor on bronchoalveolar lavage cells in hypersensitivity pneumonitis in comparison with controls and idiopathic pulmonary fibrosis. The increased expression of these three receptors may be important in the pathogenesis of hypersensitivity pneumonitis.

\section{REFERENCES}

1 Armstrong L, Foley NM, Millar AB. Inter-relationship between tumour necrosis factor-alpha (TNF- $\alpha$ ) and TNF soluble receptors in pulmonary sarcoidosis. Thorax 1999; 54: 524-530.

2 Denis M. Proinflammatory cytokines in hypersensitivity pneumonitis. Am J Respir Crit Care Med 1995; 151: 164-169.

3 Tartaglia LA, Goeddel DV, Reynolds C, et al. Stimulation of human T-cell proliferation by specific activation of the 75KDa tumor necrosis factor receptor. J Immunol 1993; 151: 4637-4641.

4 Zhou T, Mountz JD, Kimberly RP. Immunobiology of tumor necrosis factor receptor superfamily. Immunol Res 2002; 26: 323-326.

5 Agostini C, Zambello R, Sancetta R, et al. Expression of tumor necrosis factor-receptor superfamily members by lung T lymphocytes in interstitial lung disease. Am J Respir Crit Care Med 1996; 153: 1359-1367.

6 Dai H, Guzman J, Costabel U. Increased expression of apoptosis signalling receptors by alveolar macrophages in sarcoidosis. Eur Respir J 1999; 13: 1451-1454.

7 Tartaglia LA, Pennica D, Goeddel DV. Ligand passing: the 75-kDa tumor necrosis factor (TNF) receptor recruits TNF for signaling by the 55-kDa TNF receptor. J Biol Chem 1993; 268: 18542-18548.

8 Bigda J, Beletsky I, Brakebusch C, et al. Dual role of the p75 tumor necrosis factor (TNF) receptor in TNF cytotoxicity. J Exp Med 1994; 180: 445-460.

9 Daigle I, Rückert B, Schnetzler G, Simon HU. Induction of the IL-10 gene via the Fas receptor in monocytes: an antiinflammatory mechanism in the absence of apoptosis. Eur J Immunol 2000; 30: 2991-2997.

10 Kuwano K, Hara N. Signal transduction pathways of apoptosis and inflammation induced by the tumor necrosis factor receptor family. Am J Respir Cell Mol Biol 2000; 22: 147-149.

11 Luttmann W, Dauer E, Schmidt S, et al. Effect of interferon$\gamma$ and tumour necrosis factor- $\alpha$ on CD95/Fas ligandmediated apoptosis in human blood eosinophils. Scand J Immunol 2000; 51: 54-59.

12 Hara T, Tsurumi H, Takemura M, et al. Serum-soluble Fas level determines clinical symptoms and outcome of patients with aggressive Non-Hodgkin's lymphoma. Am J Hematol 2000; 64: 257-261.

13 Nagata S, Golstein P. The Fas death factor. Science 1995; 267: 1449-1455.

14 Kuwano K, Hagimono N, Kawasaki M, et al. Expression of FasL and Fas protein and their soluble form in patients with hypersensitivity pneumonitis. Int Arch Allergy Immunol 2000; 122: 209-215.
15 Brennan FM, Gibbons DL, Mitchell T, Cope AP, Maini RN, Feldman M. Enhanced expression of tumor necrosis factor mRNA and protein in mononuclear cells isolated from rheumotoid arthritis synovial joint. Eur J Immunol 1992; 22: 1907-1912

16 Amasaki Y, Kobayashi S, Takeda T, et al. Up-regulated expression of Fas antigen (CD95) by peripheral naive and memory $\mathrm{T}$ cell subsets in patients with systemic lupus erythematosus (SLE): a possible mechanism for lymphopenia. Clin Exp Immunol 1995; 99: 245-250.

17 American Thoracic Society and European Respiratory Society. American Thoracic Society/European Respiratory Society international multidisciplinary consensus classification of the idiopathic interstitial pneumonias. Am J Respir Crit Care Med 2002; 165: 277-304.

18 Costabel U, Bross KJ, Matthys H. A new method for the demonstration of surface antigens on bronchoalveolar lavage cells. Bull Eur Physiopathol Respir 1985; 21: 381-387.

19 Yamasaki $H$, Ando $M$, Brazer $W$, Center DM, Cruikshank WW. Polarized type 1 cytokine profile in bronchoalveolar lavage T cells of patients with hypersensitivity pneumonitis. J Immunol 1999; 163: 3516-3523.

20 Chen BM, Tong ZH, Nakamura S, Costabel U, Guzman J. Production of IL-12, IL-18 and TNF- $\alpha$ by alveolar macrophages in hypersensitivity pneumonitis. Sarcoidosis Vasc Diffuse Lung Dis 2004; 21: 199-203.

21 Gaede KI, Fitschen J, Ernst M, Martinet N, Schlaak M, Müller-Quernheim J. Expression of tumor necrosis factor receptors (CD120a and CD120b) on bronchoalveolar cells. Cytokine 1999; 11: 611-616.

22 Lin RH, Hwang YW, Yang BC, Lin CS. TNF receptor-2triggered apoptosis is associated with the down-regulation of Bcl-XL on activated T cells and can be prevented by CD28 costimulation. J Immunol 1997; 158: 598-603.

23 Teh HS, Seebaran A, Teh SJ. TNF receptor 2-deficient CD8 $\mathrm{T}$ cells are resistant to Fas/Fas ligand-induced cell death. J Immunol 2000; 165: 4814-4821.

24 Yang YC, Hsu TY, Chen JY, Yang CS, Lin RH. Tumor necrosis factor- $\alpha$-induced apoptosis in cord blood $\mathrm{T}$ lymphocytes: involvement of both tumor necrosis factor receptor type 1 and 2. Br J Haematol 2001; 115: 435-441.

25 Zheng LX, Fisher G, Miller RE, Peschon J, Lynch DH, Lenardo MJ. Induction of apoptosis in mature $\mathrm{T}$ cells by tumor necrosis factor. Nature 1995; 377: 348-351.

26 Dai H, Guzman J, Bauer PC, Costabel U. Elevated levels of soluble TNF receptors in bronchoalveolar lavage fluid in extrinsic allergic alveolitis. Clin Exp Allergy 1999; 29: 1209-1213.

27 Park DR, Thomsen AR, Frevert CW, et al. Fas (CD95) induces proinflammatory cytokines by human monocytes and monocyte-derived macrophages. J Immunol 2003; 170: 6209-6216.

28 Skerrett S, Martin TR, Chi EY, Peschon JJ, Mohler KM, Wilson CB. Role of the type 1 TNF receptor in lung inflammation after inhalation of endotoxin or Pseudomonas aeruginosa. Am J Physiol Lung Cell Mol Physiol 1999; 276: L715-L727.

29 Hagimoto N, Kuwano K, Miyazaki H, et al. Induction of apoptosis and pulmonary fibrosis in mice in response to ligation of Fas antigen. Am J Respir Cell Mol Biol 1997; 17: $272-278$. 